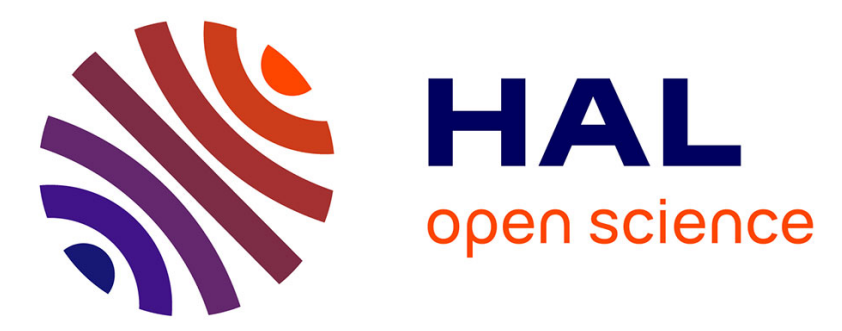

\title{
U-Joint Induced Torsional Instabilities of a Family of 3-DOF Partially-Decoupled Spherical Parallel Manipulators
}

\author{
Guanglei Wu, Stéphane Caro
}

\section{To cite this version:}

Guanglei Wu, Stéphane Caro. U-Joint Induced Torsional Instabilities of a Family of 3-DOF PartiallyDecoupled Spherical Parallel Manipulators. In: Wang D., Petuya V., Chen Y., Yu S. (eds) Recent Advances in Mechanisms, Transmissions and Applications. MeTrApp 2019. Mechanisms and Machine Science, 79, pp.332-343, 2019, 10.1007/978-981-15-0142-5_33 . hal-02404252

\section{HAL Id: hal-02404252 https://hal.science/hal-02404252}

Submitted on 11 Dec 2019

HAL is a multi-disciplinary open access archive for the deposit and dissemination of scientific research documents, whether they are published or not. The documents may come from teaching and research institutions in France or abroad, or from public or private research centers.
L'archive ouverte pluridisciplinaire HAL, est destinée au dépôt et à la diffusion de documents scientifiques de niveau recherche, publiés ou non, émanant des établissements d'enseignement et de recherche français ou étrangers, des laboratoires publics ou privés. 


\title{
U-joint Induced Torsional Instabilities of a Family of 3-DOF Partially-decoupled Spherical Parallel Manipulators
}

\author{
Guanglei $\mathrm{Wu}^{1 凶}$ and Stéphane Caro $^{2}$ \\ 1 School of Mechanical Engineering, Dalian University of Technology, 116024 Dalian, \\ China. gwu@dlut.edu.cn \\ 2 Laboratoire des Sciences du Numérique de Nantes, UMR CNRS 6004, Centre \\ National de la Recherche Scientifique (CNRS), 44321 Nantes, France. \\ stephane.caro@ls2n.fr
}

\begin{abstract}
This paper deals with the dynamic torsional stability problem of a family of partially-decoupled spherical parallel manipulators. The linearized equations of motion of the mechanical system are established to analyze the stability of the U-joint mechanism, resorting to the Floquet theory. Parametric stability charts of misalignment angles versus rotating speeds of the driving shaft are constructed to identify the unstable regions and critical shaft speeds, together with the effect of the parameters onto the manipulator stability. As a consequence, some criteria for the design and the operational speed of the manipulator, in terms of dynamic stability, are introduced.
\end{abstract}

Keywords: dynamic stability, spherical parallel manipulator, monodromy matrix, Floquet theory, torsional vibrations

\section{Introduction}

Three-degree-of-freedom (3-dof) spherical parallel manipulators (SPMs), working as robotic joints [1], orientating device [9], and devices with remote center of motion [13], have been studied in the literature for several decades. Typical symmetrical SPMs, such as $3 \mathrm{RRR}^{3}$ or $3 \mathrm{UPU}-\mathrm{S}$ ones $[3,10,20,21]$, usually have limited rotational capabilities, particularly, the torsional movement around the axis normal to the end-effector. In order to realize the unlimited rotation to extend the applications of the SPMs, an asymmetrical 2RRR-RUR-RPS SPM [24] as shown in Fig. 1(a) was proposed with a number of advantages compared to its symmetrical counterparts, such as enhanced positioning accuracy [21], infinite end-effector rotational motion [1], structural compactness and low dynamic inertia [23]. It is noted that the two actuated limbs in-parallel can be replaced with the UPU or PUU linkages [25] as displayed in Figs. 1(b) and 1(c). The common

\footnotetext{
${ }^{3} \mathrm{U}, \mathrm{P}, \mathrm{R}$ and $\mathrm{S}$ stand for the universal, prismatic, revolute and spherical joints, respectively. An underlined letter indicates an actuated joint.
} 
design of the three SPMs adopts a $U$ joint to connect the driving shaft in the center to the end-effector, ensuring an infinite torsional movement in any configuration. Henceforth, it can work as an active spherical joint or a machine tool head as shown in Fig. 2. On the other hand, the U joint suffers from one major issue, namely, it transforms a constant input speed to a periodically fluctuating one [17], which may induce vibrations and wear. Accordingly, this paper deals with the parametric torsional stability issue, due to the presence of the identical the U-joint in the three SPMs in order to lead to the full rotational motion of their end-effectors.

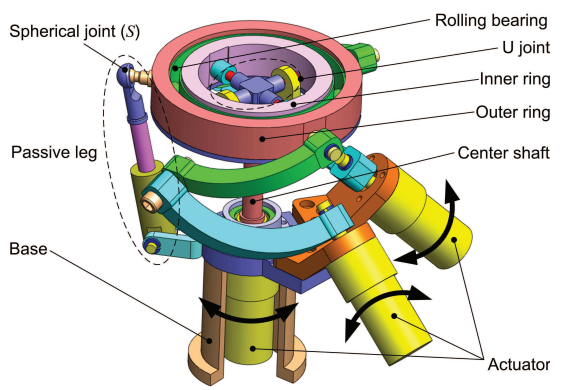

(a)

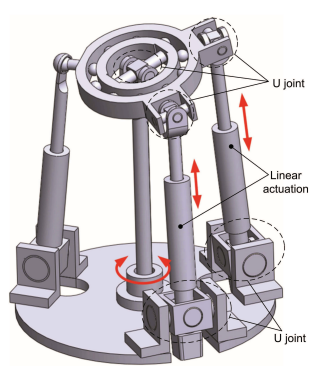

(b)

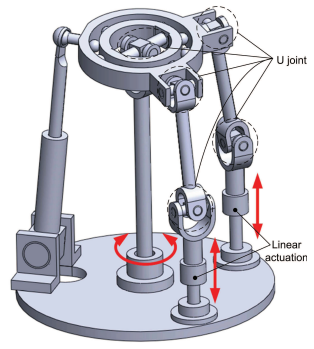

(c)

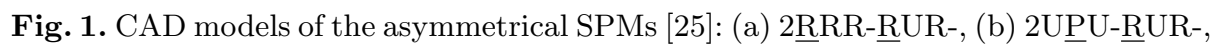
and (c) 2PUU-RUR-RPS.

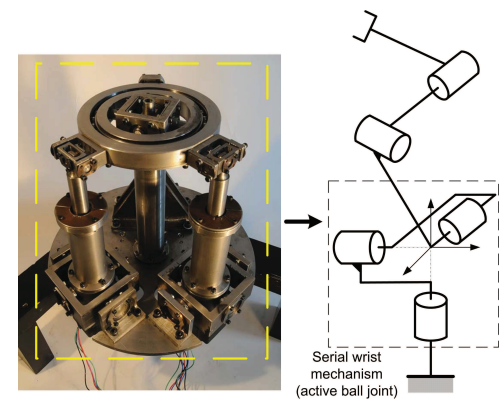

(a)

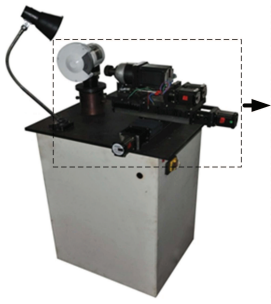

(b)

Fig. 2. Applications of the asymmetrical SPMs [25]: (a) active spherical joint; (b) drill point grinder.

To the best of the authors' knowledge, Porter [15] was the first to investigate this problem. Indeed, he expressed a single degree-of-freedom linearized model 
to plot the stability chart by using the Floquet theory $[8,12]$. Porter and Gregory [16] and Éidinov et al. [7] revisited the same problem to build a 1-dof nonlinear dynamic model and studied the stability problem by means of PoincaréLyapunov method. Later, similar modeling approaches were adopted to derive the linear or nonlinear models for the stability analysis of the $\mathrm{U}$ joint $[2,5]$. Moreover, the torsional stability of multi-shaft systems consisting of multiple shafts interconnected via Hooke's joints can also be studied using the previous various approaches [11]. According to the literature, the previous studies focus on single or multiple U-joint mechanisms. It should be noted that, a U joint working as a transmitting mechanism in parallel mechanisms has not received sufficient attention, and is the subject of this paper. As the relationship between the input and output shaft rotating speeds of the $U$ joint is periodic [18], the Floquet theory is an effective approach to analyze the stability of the mechanisms under study, where the monodromy matrix, as the fundamental matrix of a system of ordinary differential equations (ODEs) evaluated at the period of the coefficients of a system, is used for the analysis of periodic solutions. The previous work [22] has presented some preliminary results regarding to the manipulator stability. On the other hand, the vibration behaviors, such as resonance type and emanating points, are not identified. Moreover, the analysis in the previous work is carried out in a limited motion range of the vibrating resources, where larger bands of rotating speeds should be taken into consideration to analyze the influence of parameters onto the dynamic stability problem of the robotic manipulators.

This paper deals with the dynamic torsional stability problem of a family of asymmetrical spherical parallel manipulators with a U-joint transmitting the rotational motion of the end-effector. A linear model associated to the input and output shafts interconnected via a Hooke's joint is considered. Numerical study is carried out to assess the system stability and the effects of both the geometric and dynamic parameters, resorting to the Floquet theory. Parametric stability charts of misalignment angles versus rotating speeds of the driving shaft are constructed to identify the unstable regions and critical shaft speeds.

\section{SPM Architecture and the U-joint Mechanism}

The kinematic architecture of the manipulator shown in Fig. 1(a) is depicted in Fig. 3(a). The mobile platform is composed of an outer and inner rings connected to each other with a revolute joint, the revolute joint being realized with a rolling bearing. The orientation of the outer ring is controlled by two limbs in-parallel, and it is constrained by a fully passive leg that is offset from the center of the mobile platform to eliminate the rotational motion about the vertical axis. Through a universal joint, the decoupled rotation of the inner ring is generated by the center shaft, which also supports the mobile platform to improve the positioning accuracy.

Splitting the outer ring and the two parallel limbs as well as the passive one, the remaining parts of the manipulator can be equivalent to a U-joint mechanism, of which the center shaft is treated as the driving shaft and the inner ring is 
treated as a driven disk. The misalignment angle is denoted by $\beta$. The input and output angular velocities are denoted as $\Omega_{i n}$ and $\Omega_{\text {out }}$, respectively.

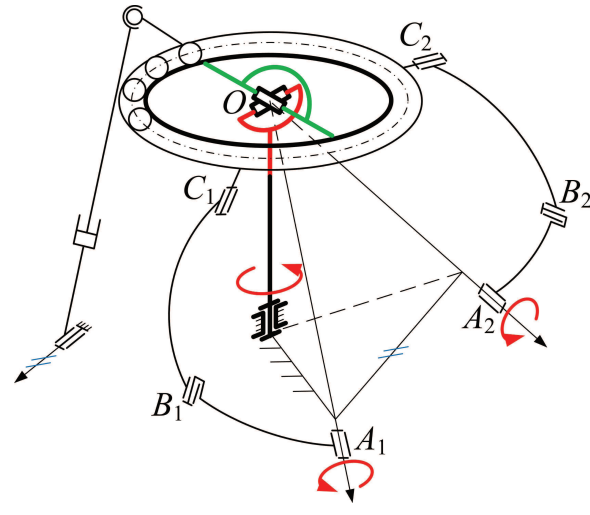

(a)

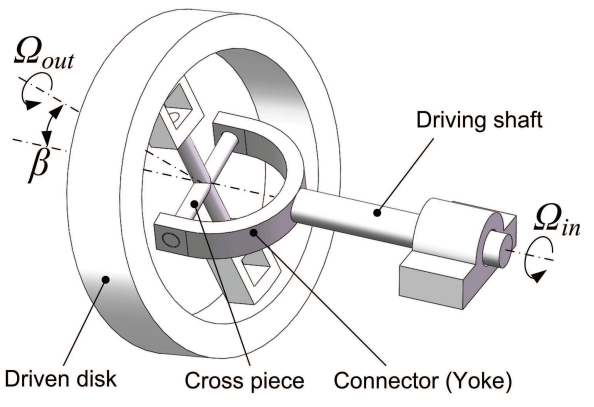

(b)

Fig. 3. Architecture of the $2 \underline{R R R-R U R-R P S ~ S P M ~(a) ~ a n d ~ i t s ~ U-j o i n t ~ t r a n s m i t t i n g ~}$ mechanism (b).

\section{Equations of Motion of Torsional Vibrations}

The equations of motion for the U-joint mechanism as shown in Fig. 3(b) are deduced via a synthetical approach $[4,22]$. In accordance, the driving shaft and the driven disk are considered as two separate parts, as displayed in Fig. 4, where the cross piece connecting the input/output elements is supposed to be as massless. Moreover, the friction is neglected.

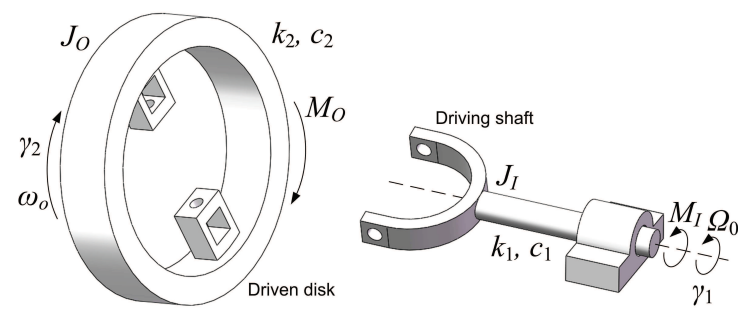

Fig. 4. The driving and driven parts of the U-joint mechanism.

Prior to dynamic modeling, the relationship between the speed of the driving part $\Omega_{i n}$ and that of the driven part $\Omega_{\text {out }}$ of the Hooke's joint, as well as the 
relationship between the input and the output torques, can be written as [18]

$$
\Omega_{\text {out }}=\Omega_{\text {in }} \cdot \eta(t), \quad M_{O}=\frac{M_{I}}{\eta(t)} ; \quad \eta(t)=\frac{\cos \beta}{1-\sin ^{2} \beta \sin ^{2}\left(\Omega_{0} t+\gamma_{1}\right)}
$$

where $\Omega_{0}$ denotes the constant velocity of the driving shaft.

Skipping the detailed modeling procedure in previous work [22], the following set of equations of motion of the U-joint mechanism, with respect to the driving and driven parts, can be expressed as:

$$
\begin{aligned}
& J_{I} \ddot{\gamma}_{1}+c_{1} \dot{\gamma}_{1}-\eta(t) c_{2} \dot{\gamma}_{2}+k_{1} \gamma_{1}-\eta(t) k_{2} \gamma_{2}=0 \\
& J_{O}\left[\ddot{\gamma}_{2}+\eta(t) \ddot{\gamma}_{1}+\dot{\eta}(t)\left(\Omega_{0}+\dot{\gamma}_{1}\right)\right]+c_{2} \dot{\gamma}_{2}+k_{2} \gamma_{2}=0
\end{aligned}
$$

with

$$
\dot{\omega}_{o}=\eta(t) \ddot{\gamma}_{1}+\dot{\eta}(t)\left(\Omega_{0}+\dot{\gamma}_{1}\right)
$$

where $\gamma_{1}$ and $\gamma_{2}$ are the rotational coordinates of $J_{I}$ and $J_{O}$, respectively, and $M_{I}$ is the reaction torque of the input part of the Hooke's joint. Moreover, $k_{1}, c_{1}$ and $k_{2}, c_{2}$ depict the torsional stiffness and viscous damper of the driving shaft and the driven disk, respectively.

By introducing the following dimensionless parameters:

$$
\Omega=\frac{\Omega_{0}}{\sqrt{k_{1} / J_{I}}}, \zeta=\frac{c_{1}}{\sqrt{k_{1} J_{I}}}, \mu=\frac{c_{2}}{c_{1}}, \nu=\frac{J_{O}}{J_{I}}
$$

Equations (2a) and (2b) are written as $[4,22]$

$$
\begin{aligned}
& \ddot{\gamma}_{1}+\frac{\zeta}{\Omega} \dot{\gamma}_{1}-\frac{\mu \zeta}{\Omega} \eta(\tau) \dot{\gamma}_{2}+\frac{1}{\Omega^{2}} \gamma_{1}-\frac{1}{\Omega^{2} \cdot \eta(\tau)} \gamma_{2}=0 \\
& \ddot{\gamma}_{2}+\left[\eta^{\prime}(\tau)-\frac{\zeta}{\Omega} \eta(\tau)\right] \dot{\gamma}_{1}+\left[\frac{\mu \zeta}{\Omega}\left(\frac{1}{\nu}+\eta^{2}(\tau)\right)\right] \dot{\gamma}_{2}+\left[\eta^{\prime \prime}(\tau)-\frac{1}{\Omega^{2}} \eta(\tau)\right] \gamma_{1} \\
& \quad+\left[\frac{1}{\Omega^{2}}\left(\frac{1}{\nu \eta(\tau)^{2}}+1\right)\right] \gamma_{2}+\eta^{\prime}(\tau)=0
\end{aligned}
$$

where $\tau=\Omega_{0} t$, and $\eta(\tau)$ is a $\pi$-periodic equation:

$$
\eta(\tau)=\eta(\pi+\tau)=\frac{\cos \beta}{1-\sin ^{2} \beta \sin ^{2} \tau}
$$

Equations (5) and (6) can be cast into a matrix form, as follows

$$
\begin{aligned}
{\left[\begin{array}{l}
\ddot{\gamma}_{1} \\
\ddot{\gamma}_{2}
\end{array}\right]+} & {\left[\begin{array}{cc}
\frac{\zeta}{\Omega} & -\frac{\mu \zeta}{\Omega} \eta(\tau) \\
\eta^{\prime}(\tau)-\frac{\zeta}{\Omega} \eta(\tau) \frac{\mu \zeta}{\Omega}\left(\frac{1}{\nu}+\eta^{2}(\tau)\right)
\end{array}\right]\left[\begin{array}{l}
\dot{\gamma}_{1} \\
\dot{\gamma}_{2}
\end{array}\right] } \\
& +\left[\begin{array}{cc}
\frac{1}{\Omega^{2}} & -\frac{1}{\Omega^{2}} \frac{1}{\eta(\tau)} \\
\eta^{\prime \prime}(\tau)-\frac{1}{\Omega^{2}} \eta(\tau) & \frac{1}{\Omega^{2}}\left(\frac{1}{\nu} \cdot \frac{1}{\eta(\tau)^{2}}+1\right)
\end{array}\right]\left[\begin{array}{l}
\gamma_{1} \\
\gamma_{2}
\end{array}\right]=\left[\begin{array}{c}
0 \\
-\eta^{\prime}(\tau)
\end{array}\right]
\end{aligned}
$$

where primes denote differentiation with respect to $\tau$, thus, Eq. (8) consists of a set of linear differential equations with $\pi$-periodic coefficients, for the torsional vibrations of the U-joint mechanism as shown in Fig. 3(b). 


\section{Dynamic Stability Analysis}

The right-hand term of Eq. (8) involves time-derived motions, which can lead to forced vibrations [14]. The scope of this study is about the self-excitation vibrations, namely, parametrically excited stability problem. Thus, the homogeneous parts of Eq. (8) should be considered sequentially to analyze the dynamic stability of the manipulator. Equation (8) can be expressed as in the following compact form:

$$
\ddot{\gamma}+\mathbf{U} \dot{\gamma}+\mathbf{V} \gamma=\mathbf{0}
$$

with

$$
\begin{aligned}
& \ddot{\gamma}=\left[\ddot{\gamma}_{1} \ddot{\gamma}_{2}\right]^{T}, \dot{\gamma}=\left[\begin{array}{ll}
\dot{\gamma}_{1} & \dot{\gamma}_{2}
\end{array}\right]^{T}, \gamma=\left[\begin{array}{ll}
\gamma_{1} & \gamma_{2}
\end{array}\right]^{T} \\
& \mathbf{U}=\left[\begin{array}{cc}
\frac{\zeta}{\Omega} & -\frac{\mu \zeta}{\Omega} \eta(\tau) \\
\eta^{\prime}(\tau)-\frac{\zeta}{\Omega} \eta(\tau) & \frac{\mu \zeta}{\Omega}\left(\frac{1}{\nu}+\eta^{2}(\tau)\right)
\end{array}\right] \\
& \mathbf{V}=\left[\begin{array}{cc}
\frac{1}{\Omega^{2}} & -\frac{1}{\Omega^{2}} \frac{1}{\eta(\tau)} \\
\eta^{\prime \prime}(\tau)-\frac{1}{\Omega^{2}} \eta(\tau) \frac{1}{\Omega^{2}}\left(\frac{1}{\nu} \cdot \frac{1}{\eta(\tau)^{2}}+1\right)
\end{array}\right]
\end{aligned}
$$

which can be represented by a state-space formulation, namely,

$$
\dot{\mathbf{x}}(t)=\mathbf{A}(t) \mathbf{x}(t) ; \quad \mathbf{x}(t)=\left[\begin{array}{c}
\gamma \\
\dot{\gamma}
\end{array}\right], \mathbf{A}(t)=\left[\begin{array}{cc}
\mathbf{0}_{2} & \mathbf{I}_{2} \\
-\mathbf{V} & -\mathbf{U}
\end{array}\right]
$$

whence $\mathbf{A}(t)$ is a $4 \times 4 \pi$-periodic state transition matrix, $\mathbf{I}$ being the identity matrix. According to Floquet theory, the solution to equation system (11) can be expressed as:

$$
\boldsymbol{\Phi}(\tau)=\mathbf{P}(\tau) e^{\tau \mathbf{R}}
$$

where $\mathbf{P}(\tau)$ is a $\pi$-periodic matrix and $\mathbf{R}$ is a constant matrix, which is related to another constant matrix $\mathbf{H}$, referred to as monodromy matrix, with $\mathbf{R}=\ln \mathbf{H} / \pi$. If the fundamental matrix is normalized so that $\mathbf{P}(0)=\mathbf{I}_{4}$, then $\mathbf{H}=\mathbf{P}(\pi)$.

The eigenvalues $\lambda_{i}=\kappa_{i}+j \sigma_{i}, i=1,2,3,4, j^{2}=-1$, of matrix $\mathbf{H}$, referred to as Floquet multipliers, govern the stability of the system. The system is asymptotically stable if and only if the real parts $\kappa_{i}$ of all the eigenvalues $\lambda_{i}$ are non-positive [6]. Here, the matrix $\mathbf{H}$ is obtained numerically with the improved Runge Kutta Method [19] with a step size equal to $10^{-6}$. The detailed calculation of the state transition matrix is given in Appendix A. As a result, the eigenvalues of matrix $\mathbf{H}$ can be calculated to assess stability of the system. The monodromy matrix method is a simple and reliable method to determine the stability of parametrically excited periodic systems.

\section{$5 \quad$ Numerical Study and Discussions}

This section is devoted to numerical stability analysis, where the stability charts are constructed on the $\Omega_{0}-\beta$ parametric planes to study the effect of parameters onto the system stability. The parameters in the analysis are given in each figure 
caption, and the ratio of the output and input dampers is set to $\mu=1$ due to the same manufacturing material.

Before presentation of the stability charts, the expected features associated to the vibration behaviors will be discussed. According to Eq. (8), the U-joint mechanism is built as a 2-dof vibrating systems, henceforth, the systems has two resonance (natural) frequencies, meaning that the system will vibrate if it is excited by some periodical phenomena. Setting $\zeta=0, \beta=0, \Omega=1$, these frequencies are calculated as

$$
\omega_{1(2)}=\sqrt{\frac{k_{1}}{J_{I}}\left(1-\frac{-1 \pm \sqrt{4 \nu^{2}+1}}{2 \nu}\right)}
$$

If no damping is present, the parametric resonance occurs when the frequency $\omega_{j}, j=1,2$, is equal to the product of an integer and the rotating speed $\Omega_{0}$ of the driving shaft, thus, the parametric resonances are expected to emanate from [14]

$$
\Omega_{j, k}=\frac{\omega_{j}}{k} ; \quad j=1,2, \quad k=1,2,3, \ldots
$$

and the parametric combination resonances based on the sum or difference of the two frequencies [14] are expected to emanate from

$$
\Omega_{k}^{+}=\frac{\omega_{1}+\omega_{2}}{2 k}, \quad \Omega_{k}^{-}=\frac{\omega_{2}-\omega_{1}}{2 k} ; \quad k=1,2,3, \ldots
$$

Sequentially, the critical rotating speeds of the driving shaft can be identified from Eqs. (14) and (15).

Figure 5 depicts the stability chart $\Omega_{0}-\beta$ to detect the instabilities of the Ujoint mechanism with the prescribed parameters, where the circle-marked zones and solid markers represent the unstable parametric regions and the emanation points, respectively. It is seen that parametric resonances are located in the unstable regions. It should be noted that as long as the the rotating speed $\Omega_{0}$ of the driving shaft is lower than $7 \pi \mathrm{rad} / \mathrm{s}$, this system is stable for any misalignment angle $\beta$ between 0 and $30^{\circ}$. On the contrary, the mechanism will be stable for any driving shaft speed smaller than $50 \pi \mathrm{rad} / \mathrm{s}$ as long as the misalignment angle $\beta$ is lower than $5^{\circ}$.

The effects of the parameters on the manipulator stability are depicted in Figs. 6 to 9, where the marked areas stand for the unstable regions. Moreover, the analysis is carried out with the identical parameters but except one, for a comparative study.

Figure 6 depicts the effect of the torsional stiffness of the driving shaft on the stability, by comparing three different values of $k_{1}$. The manipulator is no longer stable if $\Omega_{0}>36 \pi \mathrm{rad} / \mathrm{s}$ when $k_{1}=5 \mathrm{Nm} / \mathrm{rad}$. With lower stiffness, the critical rotating speed of the driving shaft is in a smaller threshold, according to the comparison between the cases of $k_{1}=10 \mathrm{Nm} / \mathrm{rad}$ and $k_{1}=5 \mathrm{Nm} / \mathrm{rad}$. With the increasing stiffness, the stable region becomes wider. It is noteworthy that the higher the torsional stiffness of the input shaft, the more stable the manipulator. 


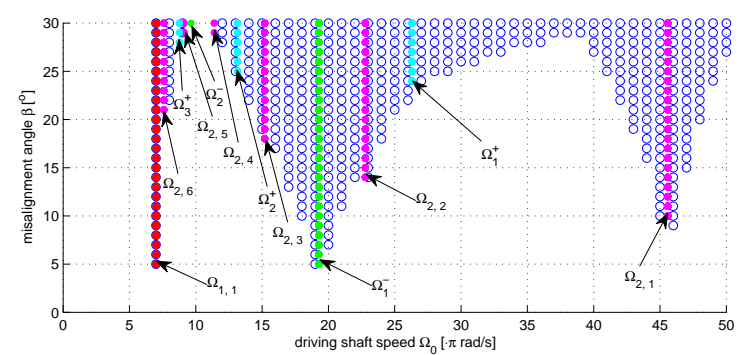

Fig. 5. Stability chart: unstable regions (circle point) with parameters $k_{1}=10 \mathrm{Nm} / \mathrm{rad}$, $\nu=10, J_{I}=0.001 \mathrm{~kg} \cdot \mathrm{m}^{2}, c_{1}=0.001 \mathrm{Nm} /(\mathrm{rad} / \mathrm{s})$.

Figure 7 shows the effect of the damping ratio on the system stability, from which it is seen that the unstable regions are almost coincident with the dampers that are in the range of the damping ratio of common materials, with a few exceptions when $c_{1}=0.0005 \mathrm{Nm} /(\mathrm{rad} / \mathrm{s})$. This means that the influence of the damping ratio to the system stability is insignificant and negligible.

Similarly, Fig. 8 depicts the effect of the mass moment of inertia of the driving shaft to the parametric stability. When the inertia reduces to $J_{I}=0.0005 \mathrm{~kg} \cdot \mathrm{m}^{2}$, the range of the critical misalignment angle and the shaft rotating speed become larger, together with the increasing threshold. Conversely, the increased inertia speeds up instabilities of the system.

Figure 9 displays the unstable regions of the manipulator with different ratios of the mass moment of inertia of the driven disk and the driving shaft, which shows that the influence of this parameter is not so significant. It is noteworthy that the smallest ratio can ensure the largest critical rotating speed of the driving shaft versus the misalignment angle.

An observation from Figs. 6 to 9 shows that all the parameters except the damping ratio heavily influence the stability of the manipulator, namely, higher actuation stiffness, lower inertia of the driving shaft and larger inertia ratio of the driven and driving parts being helpful for the dynamic stability. Moreover, an efficient approach to overcome the instability problem of the manipulator with higher rotating speed of driving shaft is to improve the actuation stiffness, for a lightweight design and high stiffness of the end-effector constrained by the driving shaft. All the stability charts show the critical rotating speeds of the driving shaft versus the misalignment angles influenced by both geometric and dynamic parameters. Accordingly, those charts can be used to find the optimum design parameters of the mechanism while guaranteeing its dynamic stability for a range of driving shaft speed.

Compared to the dynamic model in Ref. [4], the term of stiffness ratio of the output and input elements in Eq. (8) is related by the U-joint relationship, instead of a designated parameter, which can simplify the dynamic model and better characterize the manipulator stability. Moreover, the stability charts of previous numerical results $[4,14]$ are represented by dimensionless param- 

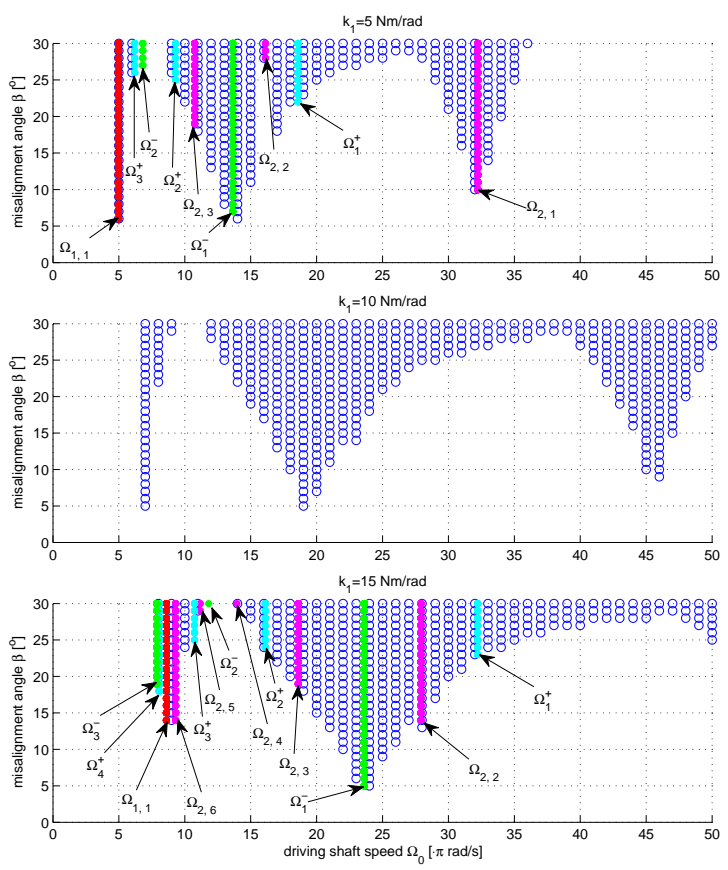

Fig. 6. Stability Chart: effects of the driving shaft stiffness $k_{1}$ with parameters $\nu=10$, $J_{I}=0.001 \mathrm{~kg} \cdot \mathrm{m}^{2}, c_{1}=0.001 \mathrm{Nm} /(\mathrm{rad} / \mathrm{s})$.

eters/ratios, thus, the relationship of the misalignment angles versus rotating speeds of the driving shaft in this work can be characterized intuitively.

\section{Conclusions}

This paper presented the dynamic torsional stability analysis of a family of 3dof partially-decoupled spherical parallel manipulators due to the transmitting universal joint. The linearized equations of motion of the system were built to study the torsional stability problem of the manipulator due to the nonlinear input-output transmission of the U-joint, where the relationship of the stiffness between the input and output elements is involved to characterize the manipulator stability, compared to the existing model.

The approach used to analyze the torsional stability problem was numerically illustrated, wherein the unstable regions are presented graphically as well as the parametric resonances. Parametric stability charts of misalignment angles versus rotating speeds of the driving shaft are constructed to identify the unstable regions and critical shaft speeds. Results show that higher actuation stiffness, lower inertia of the driving shaft and larger inertia ratio of the driven and driving parts can ensure the manipulator stable. Moreover, an efficient approach to overcome the instability problem of the manipulator with higher rotating speed 


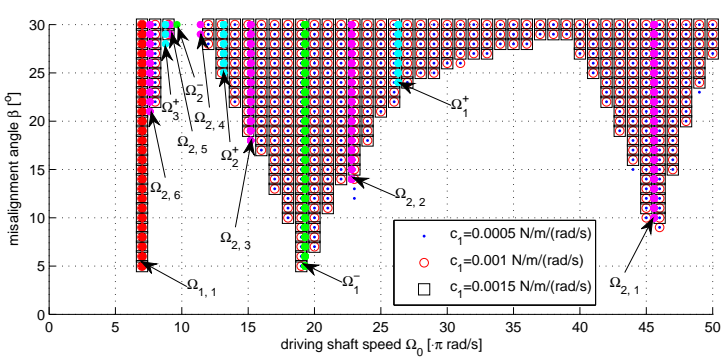

Fig. 7. Stability Chart: effects of the damping ratio $c_{1}$ with parameters $k_{1}=$ $10 \mathrm{Nm} / \mathrm{rad}, \nu=10, J_{I}=0.001 \mathrm{~kg} \cdot \mathrm{m}^{2}$.
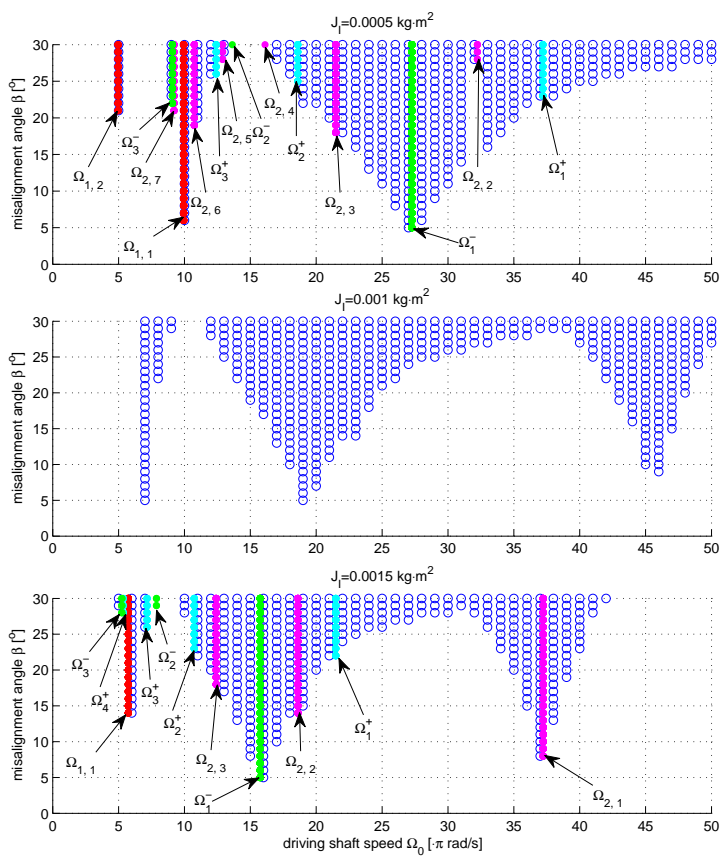

Fig. 8. Stability Chart: effects of the inertia $J_{I}$ with parameters $k_{1}=10 \mathrm{Nm} / \mathrm{rad}$, $\nu=10, c_{1}=0.001 \mathrm{Nm} /(\mathrm{rad} / \mathrm{s})$. 

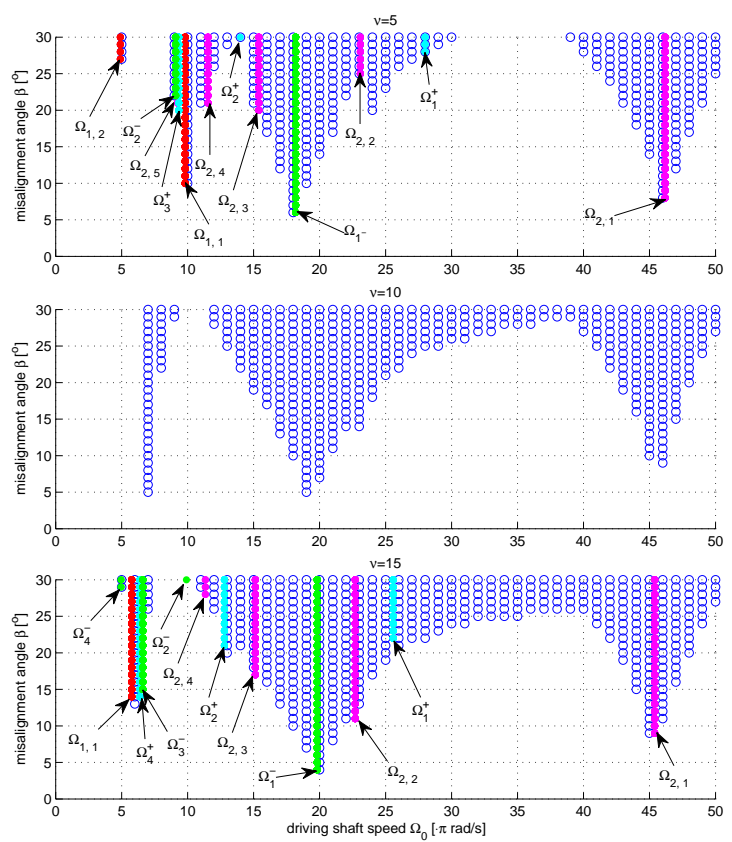

Fig. 9. Stability Chart: effects of the inertia ratio $\nu$ with parameters $k_{1}=10 \mathrm{Nm} / \mathrm{rad}$, $J_{I}=0.001 \mathrm{~kg} \cdot \mathrm{m}^{2}, c_{1}=0.001 \mathrm{Nm} /(\mathrm{rad} / \mathrm{s})$.

of driving shaft is to improve the actuation stiffness, for a lightweight design and high stiffness of the end-effector constrained by the driving shaft. The obtained charts can be used to find the optimum design parameters of the mechanism while guaranteeing its dynamic stability for a range of driving shaft speed.

\section{Acknowledgement}

The supports from the Fundamental Research Funds for the Central Universities (No.DUT19JC25), the Natural Science Foundation and the Doctoral Start up Foundation of Liaoning Province (Nos. 20180520028, 20170520134) are gratefully appreciated.

\section{References}

1. Asada, H., Granito, J.: Kinematic and static characterization of wrist joints and their optimal design. In: IEEE Int. Conf. Robot. Autom., pp. 244-250 (1985)

2. Asokanthan, S.F., Hwang, M.C.: Torsional instabilities in a system incorporating a hooke's joint. J. Vib. Acoust. 118(3), 83-91 (1996)

3. Bai, S.: Optimum design of spherical parallel manipulator for a prescribed workspace. Mech. Mach. Theory 45(2), 200-211 (2010) 
4. Bulut, G., Parlar, Z.: Dynamic stability of a shaft system connected through a hooke's joint. Mech. Mach. Theory 46(11), 1689-1695 (2011)

5. Chang, S.I.: Torsional instabilities and non-linear oscillation of a system incorporating a hooke's joint. J. Sound Vib. 229(4), 993-1002 (2000)

6. Chicone, C.: Ordinary Differential Equations with Applications, chap. 2. Springer, New York, NY (2006)

7. Éidinov, M.S., Nyrko, V.A., Éidinov, R.M., Gashukov, V.S.: Torsional vibrations of a system with hooke's joint. Soviet Appl. Mech. 12(3), 291-298 (1976)

8. Floquet, G.: Sur les équations différentielles linéaires à coefficients périodiques. Annales de l'École Normale Supérieure 12, 47-88 (1883)

9. Gosselin, C., Hamel, J.: The Agile Eye: a high-performance three-degree-of-freedom camera-orienting device. In: IEEE Inter. Conf. Robot. Autom., pp. 781-786 (1994)

10. Kong, X., Gosselin, C.: Type synthesis of 3-dof spherical parallel manipulators based on screw theory. ASME J. Mech. Des. 126(1), 101-108 (2004)

11. Kotera, T.: Instability of torsional vibrations of a system with a cardan joint. Memoirs of the Faculty of Engineering Kobe University 26, 19-30 (1980)

12. Kuchment, P.A.: Floquet Theory For Partial Differential Equations, chap. 4. Birkhauser Verlag (1993)

13. Li, T., Payandeh, S.: Design of spherical parallel mechanisms for application to laparoscopic surgery. Robotica 20(2), 133-138 (2002)

14. Mazzei Jr, A.J., Argento, A., Scott, R.A.: Dynamic stability of a rotating shaft driven through a universal joint. J. Sound Vib. 222(1), 19-47 (1999)

15. Porter, B.: A theoretical analysis of the torsional oscillation of a system incorporating a hooke's joint. ARCHIVE J. Mech. Eng. Sci. 3(4), 324-329 (1961)

16. Porter, B., Gregory, R.W.: Non-linear torsional oscillation of a system incorporating a hooke's joint. ARCHIVE J. Mech. Eng. Sci. 5(2), 191-209 (1963)

17. Rosenberg, R.M.: On the dynamical behavior of rotating shafts driven by universal (hooke) couplings. ASME J. Appl. Mech. 25(1), 47-51 (1958)

18. Seherr-Thoss, H.C., Schmelz, F., Aucktor, E.: Universal Joints and Driveshafts: Analysis, Design, Applications. Springer Berlin Heidelberg (2006)

19. Szymkiewicz, R.: Numerical Solution of Ordinary Differential Equations, chap. 4. Academic Press (1971)

20. Urízar, M., Petuya, V., Altuzarra, O., Diez, M., Hernández, A.: Non-singular transitions based design methodology for parallel manipulators. Mech. Mach. Theory 91, 168-186 (2015)

21. Wu, G., Bai, S., Kepler, J.: Mobile platform center shift in spherical parallel manipulators with flexible limbs. Mech. Mach. Theory 75, 12-26 (2014)

22. Wu, G., Caro, S.: Torsional stability of a U-joint based parallel wrist mechanism featuring infinite torsion. In: 22nd CISM IFToMM Symposium on Robot Design, Dynamics and Control, pp. 147-154. Rennes, France (2018)

23. Wu, G., Caro, S., Bai, S., Kepler, J.: Dynamic modeling and design optimization of a 3-DOF spherical parallel manipulator. Robot. Auto. Syst. 62, 1377-1386 (2014)

24. Wu, G., Caro, S., Wang, J.: Design and transmission analysis of an asymmetrical spherical parallel manipulator. Mech. Mach. Theory 94, 119-131 (2015)

25. Wu, G., Zou, P.: Comparison of 3-dof asymmetrical spherical parallel manipulators with respect to motion/force transmission and stiffness. Mech. Mach. Theory 105, 369-387 (2016) 